\title{
NOTE ON THE CONSTRUCTION OF TABLES OF LINEAR FORMS*
}

BY D. N. LEHMER

Certain theorems concerning the linear forms that go with a given quadratic residue seem not to have been noticed, or if noticed, not to have been appreciated by those who have constructed tables of divisors of quadratic forms. In connection with work on factor stencils, the author has found it necessary to extend these tables of linear forms, and the construction and checking has been much facilitated and rendered more accurate by the simple observation that if $a$ and $b$ are two entries in such a table so also is the product $a b$. In particular, the powers of any entry are also in the list of entries. This indicates a very rapid method of computation that is well adapted to a computing machine. If $R$ is of the form $4 n+1$, then in the table for $R$, if an entry $a$ appears so also will $-a$. This furnishes an important check on the accuracy of the work. The numerous errors in Legendre's tables could not have escaped detection if this check had been employed.

The table for $-R$ may be easily written down without computation from the table for $+R$ and conversely, when it is observed that entries of the form $4 n+1$ appear in the same place in both tables while entries of the form $4 n-1$ which appear in one table must not appear in the other.

Availing himself of these theorems and checks, the author has already extended the tables of forms as far as +300 and -300 . The tables for +58 and -58 are subjoined to indicate the form used and to illustrate the method of construction and the checks. Thus the first table shows that the forms for +58 are $232 n+1,3,7,9,11,19,21$, $23,25,27,33, \cdots$, these entries being indicated by 1's. Numbers not prime to the modulus 58 are indicated by

* Presented to the Society, San Francisco Section, June 19, 1925. 
circles. Since $(58 / 3)=1$, we can start with the entry 3 in this table and enter the powers of 3 , casting out multiples of 232 as they arise. This process gives all the entries for this table. The entries may appear in several cycles, of course, instead of one as in this case.

The table for $-R$ is now built from this by setting over into it the entries of the form $4 n+1$ as they stand, such as $1,9,21,25,33,37, \ldots$, and then filling in the blanks in the first table which correspond to numbers of the form $4 n-1$, such as $15,31,35,39,47, \ldots$, avoiding the numbers marked with circles. It is then carefully verified that the table for +58 reads backwards and forwards the same, while in the table for -58 the blanks at one end of the table match with the 1's at the other. The theorems upon which these checks are based are easily deduced from Jacobi's extension of Legendre's Law of Reciprocity.

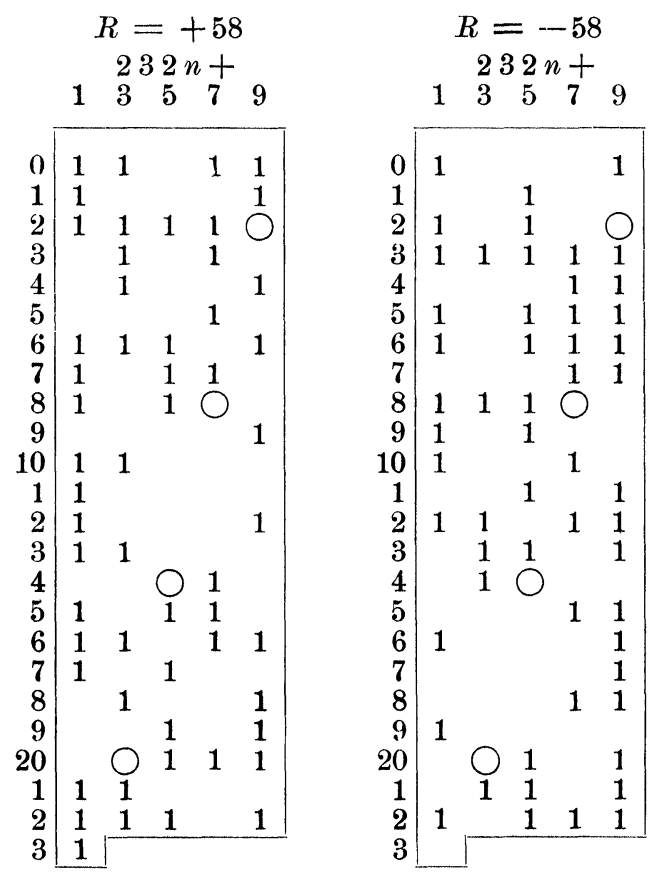

The University of California 